\title{
A numerical study of the scale effects affecting the evolution and sediment entrainment capacity of a gravity current, propagating over a loose bed containing large-scale roughness elements
}

\author{
T. Tokyay \& G. Constantinescu \\ Department of Civil and Environmental Engineering, \\ The University of Iowa, USA
}

\begin{abstract}
When compositional gravity currents propagate over a loose bed, they can entrain, carry, and deposit large quantities of sediment over considerable distances from the entrainment location. The capacity of compositional gravity currents to entrain sediment of various sizes from a loose bed containing largescale roughness elements in the form of two-dimensional (2D) ribs is investigated using high-resolution Large Eddy Simulation (LES). The compositional gravity current is produced by the instantaneous release of a large volume of heavier lock fluid in a straight open channel. The present study focuses on the influence of the Reynolds number and the presence of 2D ribs on the evolution of the lock-exchange gravity current and on the distributions of the bed friction velocity. At the higher Reynolds number considered in the present investigation, the structure of the gravity current is closer to the inviscid state often assumed in simplified models.
\end{abstract}

Keywords: gravity currents, sediment entrainment, Large Eddy Simulation, large-scale roughness.

\section{Introduction}

Gravity currents are mainly horizontal flows moving under the influence of gravity and generated by predominantly horizontal density differences within a 
fluid or between two fluids. Predicting and understanding the evolution of gravity currents is of considerable interest to many applications in geophysics, in particular due to their impact on the environment. In the case in which they propagate over a loose bed (e.g., in rivers, lakes or oceans), compositional gravity currents can entrain substantial amounts of sediment and induce the formation of a turbidity gravity current. The turbulent motions within the body of the current detach particles from the bed and entrain them into the channel to form a turbidity current. Additionally, in most cases the loose bed over which the gravity current propagates is not smooth and flat. The presence of large-scale roughness elements can significantly modify the capacity of the compositional gravity current to entrain sediment with respect to the case in which the bed is flat. For example, erosion by gravity currents is one of the main causes for the formation of submarine canyons.

Detailed measurements of the velocity and density fields within the gravity current are seldom available from experimental studies. In many applications involving turbidity currents or compositional currents propagating over loose beds, information on the spatial and temporal distributions of the bed friction velocity are essential to determine the amount of sediment entrained and carried by the current. High-resolution three-dimensional numerical simulations using eddy-resolving techniques like Direct Numerical Simulation (e.g., see Hartel et al., [3]) and Large Eddy Simulation (e.g., Ooi et al., [6]) have the advantage that can predict the spatial and temporal evolution of these variables. In particular, LES allows performing simulations at Reynolds numbers that are closer to the range typically encountered in practical applications. In many applications in river and ocean engineering, the Reynolds numbers are very high and the structure of the gravity current approaches the inviscid limit. Laboratory studies are typically conducted at much lower Reynolds numbers.

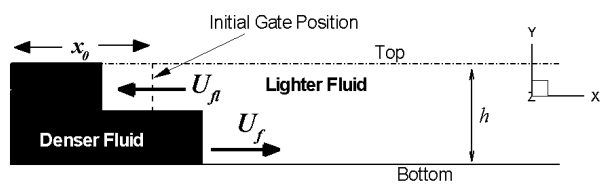

Figure 1: $\quad$ Sketch of a lock exchange flow in a channel after the gate was removed with associated front velocities of the heavier $\left(U_{f}\right)$ and lighter $\left(U_{f l}\right)$ currents.

In this work we investigate the effect of the Reynolds number on the evolution and capacity to entrain sediment of a lock-exchange compositional Boussinesq gravity current propagating over a flat bed containing an array of 2D ribs. We are also studying how the capacity of the gravity current to entrain sediment changes with respect to the case when the gravity current propagates over a flat bed.

As a result of the density differences between the lock fluid and the surrounding fluid and of the hydrostatic pressure differences, the heavy fluid starts spreading underneath the lighter fluid, as shown in Figure 1. In the set up 
considered in this study, the gravity current containing lock fluid propagates in a rectangular horizontal plane channel. We consider the case in which the initial volume of the release (heavier lock fluid) is high $\left(\mathrm{x}_{0}>>\mathrm{H}\right)$ and occupies the whole depth of the channel (full-release case). The channel is long enough to avoid interactions of the gravity current with the end walls during the simulation time. Thus, this paper considers only the evolution of a gravity current during the slumping phase. The wavelength of the square ribs is $3 \mathrm{H}$ and their height is $0.15 \mathrm{H}$ (see Figure 2), where $\mathrm{H}$ is the channel depth. The changes in the structure of the gravity current and its capacity to entrain sediment are investigated between $\mathrm{Re}=48,000 \quad\left(\mathrm{Re}=\mathrm{u}_{\mathrm{b}} \mathrm{H} / \mathrm{v}\right.$, where $v$ is the molecular viscosity, $u_{b}=\sqrt{g^{\prime} H}$ is the buoyancy velocity and $g^{\prime}$ is the reduced gravity), in the range at which most laboratory experiments are conducted, and $\mathrm{Re}=10^{6}$, closer to field conditions.

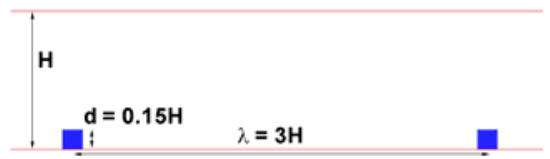

Figure 2: $\quad$ Sketch showing dimensions and spacing of the $2 \mathrm{D}$ ribs on the bottom surface.

\section{Numerical model}

A finite-volume LES code is used to solve the governing equations on nonuniform Cartesian meshes. A semi-implicit iterative method that employs a staggered conservative space-time discretization is used to advance the equations in time while ensuring second order accuracy in both space and time. A Poisson equation is solved for the pressure using multigrid. The algorithm discretely conserves energy, which allows obtaining solutions at high Reynolds numbers without artificial damping. A dynamic Smagorinsky model is used to estimate the subgrid-scale viscosity and diffusivity. All operators are discretized using central discretizations, except the convective term in the advection-diffusion equation solved for the concentration for which the QUICK scheme is used. Detailed validation of the code for 3-D LES simulations of cavity flows with or without an incoming turbulent flow is described in Chang et al. [1]. The same code was successfully used by Chang et al. [2] to predict ejection of buoyant pollutants from bottom channel cavities and by Ooi et al. [6] to predict the evolution of intrusive gravity currents.

The density difference between the lock fluid and the ambient fluid is small enough to use the Boussinesq approximation. The Navier-Stokes equations and the advection-diffusion equation for the concentration are made dimensionless using the channel depth, $\mathrm{H}$, and the buoyancy velocity, $\mathrm{u}_{\mathrm{b}}$. The time scale used in the discussion of the results is $t_{0}=H / u_{b}$. The non-dimensional concentration is defined as $C=\left(\bar{C}-\bar{C}_{\min }\right) /\left(\bar{C}_{\max }-\bar{C}_{\min }\right)$, where $\bar{C}_{\max }, \bar{C}_{\min }$ represent the 
maximum (lock fluid) and minimum (ambient fluid) concentrations in the domain and $\bar{C}$ is the dimensional concentration. The lock gate is positioned in the middle of the computational domain $(\mathrm{x} / \mathrm{H}=0.0)$. The top and bottom surfaces are simulated as no-slip smooth walls. The flow is assumed to be periodic in the spanwise direction (z). A zero normal gradient boundary condition is assumed for the concentration at the top, bottom and at the two end boundaries. All simulations discussed in this paper were conducted with a value of the viscous Schmidt number of 600 corresponding to saline water. No assumptions are needed on the value of the turbulent Schmidt number as the dynamic procedure (Chang et al. [1]) directly estimates the value of the subgrid-scale diffusivity based on the resolved velocity and concentration fields. The flow field was initialized with the fluid at rest.

The length of the computational domain in the streamwise and spanwise directions was $40 \mathrm{H}$ and $\mathrm{H}$, respectively. The mesh contained over 40 million cells and the mesh spacing in the wall normal direction was sufficiently small to resolve the viscous boundary layer (no wall functions were used) in all the simulations. The time step was $0.001 \mathrm{t}_{0}$.

To maintain the anti-symmetry of the forward and backward propagating currents containing lock and ambient fluid, respectively, the roughness elements were placed on the bottom surface in the region with $\mathrm{x} / \mathrm{H}>0$ and on the top surface in the region with $\mathrm{x} / \mathrm{H}<0$. This allows analyzing only the evolution of the forward propagating current containing heavier lock fluid. In the case in which 2D ribs were present the evolution of the gravity current is analyzed for $t<50 t_{0}$. During this period the interaction of the gravity currents containing lock and ambient fluid with the end walls is negligible.

The two simulations conducted with a flat bed are denoted Flat_LR $(\operatorname{Re}=48,000)$ and Flat HR $\left(\operatorname{Re}=10^{6}\right)$, respectively. The simulations conducted with an array of equally-spaced $2 \mathrm{D}$ ribs are denoted Ribs_LR $(\mathrm{Re}=48,000)$ and Ribs_HR $\left(\mathrm{Re}=10^{6}\right)$, respectively.

At both Reynolds numbers, the gravity current and the flow in the region behind the front is highly turbulent, similar to gravity currents of practical relevance in engineering and geophysics. We consider the gravity current is highly turbulent if velocity spectra contain a clear inertial range as the upstream part of the gravity current is convected over streamwise locations situated at a sufficient distance from the lock gate ( $\cong 4 H$ in our simulations).

\section{Results and discussion}

The presence of the ribs induces significant changes in the structure of the gravity current. For example, Figure 3 compares the distributions of the spanwise-averaged velocity magnitude inside the forward propagating current between the high Reynolds number simulations $\left(\mathrm{Re}=10^{6}\right)$ at $\mathrm{t}=32 \mathrm{t}_{0}$. In the flat bed case (Flat_HR) the velocity magnitude in the tail of the gravity current increases monotonically with the distance from the lock-gate position $(\mathrm{x} / \mathrm{H}=0.0)$ until the start of the dissipative wake region $(\mathrm{x} / \mathrm{H} \sim 14)$. In contrast to this, in the case in which 2D ribs are present (Ribs_HR), the velocity magnitude is strongly 
amplified as the lock fluid inside the gravity current is convected over the top of the rib. The amplification is the highest in the jet-like flow that forms as the heavier gravity current fluid separates from the top of the rib. The jet-like flow reattaches some distance downstream of each rib $(\sim 0.5 \mathrm{H})$, at which point the high-speed jet-like flow becomes parallel to the bed. The velocity magnitude continues to be high in the near-bed region until the presence of the next rib is felt by the gravity current. As this happens, the flow inside the gravity current starts decelerating again. Observe also that the front of the gravity current in the Ribs_HR simulation is situated about $2.5 \mathrm{H}$ behind the front position in the Flat_HR simulation. This is expected, as the presence of large-scale roughness elements at the bed introduces an additional drag force that slows down the current with respect to the case when it propagates over a flat bed.

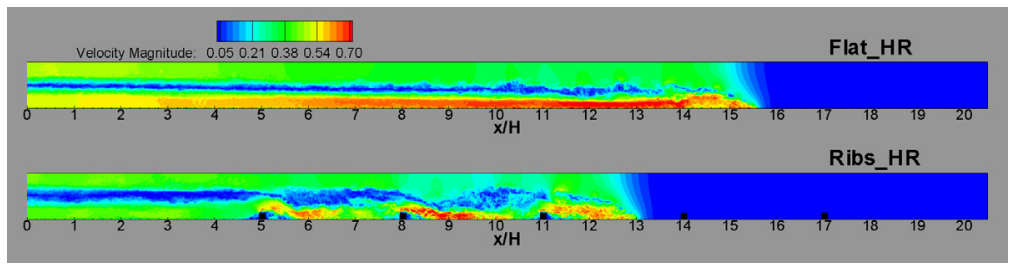

Figure 3: Distributions of the spanwise-averaged velocity magnitude at $\mathrm{t}=30 \mathrm{t}_{0}$ in the Flat_HR (top) and Ribs_HR (bottom) simulations.

Shin et al. [7] performed full-depth lock-release experiments with high Reynolds number gravity currents propagating over a flat bed. They found that the front velocity during the slumping phase was consistent with Benjamin's half-depth solution $\left(\mathrm{U}_{\mathrm{f}} / \mathrm{u}_{\mathrm{b}}=0.5\right)$. Several other experiments (e.g., Keulegan [5]) have shown that the non-dimensional front velocity of full-depth lock-release Boussinesq currents during the slumping phase increases with the Reynolds number and eventually approaches 0.5 . Our 3D LES simulations of gravity currents propagating over a flat bed confirm this trend $\left(\mathrm{U}_{\mathrm{f}} / \mathrm{u}_{\mathrm{b}}=0.49\right.$ for the Flat_HR simulation at $\left.\mathrm{Re}=10^{6}\right)$. A very good agreement between the predicted $\left(\mathrm{U}_{\mathrm{f}} / \overline{\mathrm{u}}_{\mathrm{b}}=0.45\right.$ for Flat_LR simulation $)$ and measured $\left(\mathrm{U}_{\mathrm{f}} / \mathrm{u}_{\mathrm{b}} \sim 0.45\right)$ non-dimensional front velocities is observed at $\mathrm{Re}=48,000$.

As expected, and consistent with the results in Figure 3, the presence of the ribs slows down the propagation of the front of the current. Figure 4 compares the trajectories of the front, $\mathrm{x}_{\mathrm{f}} / \mathrm{H}$, in the Ribs_HR and Flat_HR simulations. The trajectories are very close until the front of the current approaches the first bottom-attached rib in the simulation containing the ribs. Then, the two trajectories start diverging. As expected, the slope of the front trajectory in the Flat_HR simulation can be considered constant starting at the end of the acceleration phase $\left(\mathrm{t} \sim 3 \mathrm{t}_{0}\right)$. As the front was situated at more than $2 \mathrm{H}$ from the end-wall during the time interval the simulation was run, the gravity current remained in the slumping phase $\left(\mathrm{U}_{\mathrm{f}} / \mathrm{u}_{\mathrm{b}}=0.49\right)$.

The slope of the front trajectory in the Ribs_HR simulation shows a larger variation in time after the front approaches the first rib $\left(t \sim 10 t_{0}\right)$. This is mainly 
because the front is decelerating as it approaches the upstream face of each rib due to the adverse pressure gradients induced by the rib obstacle. Moreover, the velocity in the head region has to further decrease as the head rises above the top of the rib. During this time, the potential energy increases at the expense of the head losing some of its kinetic energy. Then, the front velocity increases as the head is projected downwards toward the bed. Still, the results in Figure 4 clearly show the mean slope of the trajectory can be considered in a good approximation constant for $\mathrm{t}>15 \mathrm{t}_{0}$. This means that during the slumping phase the front velocity of a gravity current propagating over a surface containing $2 \mathrm{D}$ ribs is approximately constant. The value inferred from the Ribs_HR simulation is $\mathrm{U}_{\mathrm{f}} / \mathrm{u}_{\mathrm{b}}=0.36$, which corresponds to a $27 \%$ reduction with respect to the flat-bed case. The mean front velocity predicted during the slumping phase is 0.34 for the Ribs_LR simulation and 0.45 for the Flat_LR simulation. Thus, in the lower Reynolds number simulations the reduction in the front velocity is close to $24 \%$.

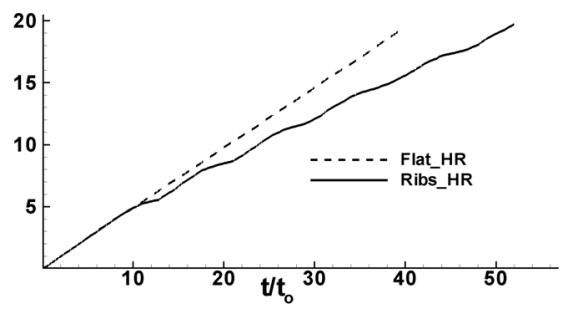

Figure 4: $\quad$ Time history of the front-position $\mathrm{x}_{\mathrm{f}} / \mathrm{H}$ for the Flat HR (dashed line) and Ribs_HR (solid line) simulations.

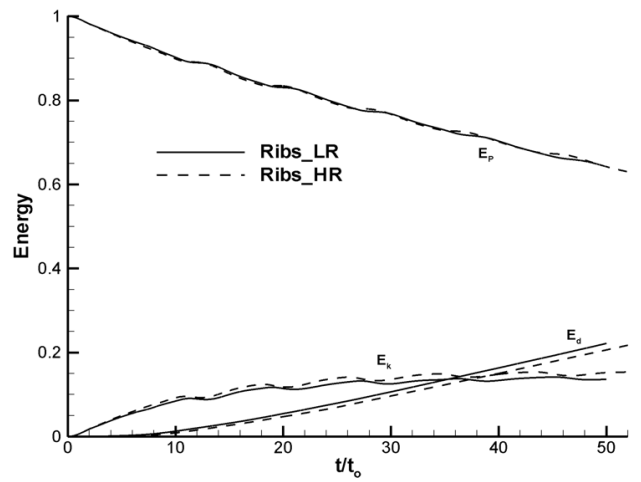

Figure 5: Time history of the potential energy, $E_{p}$, kinetic energy, $E_{k}$, and integral of the total dissipation, $\mathrm{E}_{\mathrm{d}}$, in the Ribs_LH (dashed line) and Ribs_HR (solid line) simulations.

Analysis of the global energy balance for the Ribs_LR and Ribs_HR simulations (Figure 5) allows comparing scale effects in the temporal variations of the kinetic energy, $E_{k}$, potential energy, $E_{p}$, and of the integral in time of the 
total dissipation rate integrated over the whole domain $(\varepsilon), E_{d}(t)=\int_{0}^{t} \varepsilon(\tau) d \tau$. In the case of compositional gravity currents (e.g., see Ooi et al. [6]) the sum of these three terms should be constant in time $\left(E_{k}+E_{p}+E_{d}=\right.$ constant $)$. Scale effects are negligible for the variation of the potential energy. The effect of the increase in the Reynolds number is to increase the front speed and the kinetic energy. Most of the increase in the kinetic energy is compensated by a decrease in the dissipation term $E_{d}$. This is because as the Reynolds number increases, the local dissipation rate that is proportional to the molecular viscosity $(1 / \mathrm{Re})$ is expected to decrease despite the fact that the velocity gradients within the turbulent gravity current are larger. The regions of relative minima observed in the temporal variation of $\mathrm{E}_{\mathrm{k}}$ correspond to the time intervals when the front starts interacting with the upstream face of the rib and the head is convected over the top of the rib.

The effect of the increase of the Reynolds number on the temporal evolutions of $E_{k}, E_{p}$ and $E_{d}$ in the flat-bed simulations is qualitatively similar. The only difference is the fact that the relative increase in $E_{k}$ and the corresponding decrease in $E_{d}$ are larger compared to the case when the bed surface contains ribs. Thus, the importance of scale effects decreases in the case in which the bed contains large-scale roughness elements.

The bed friction velocity distributions are needed to estimate the amount of sediment entrained from the bed by a compositional gravity current propagating over a loose bed. This is because most sediment entrainment formulae predict the entrainment rate function of the bed-friction velocity. For example, in the case of Van Rijn's [8] model the local pick up rate, $\mathrm{P}$, is proportional to the difference between the actual bed friction velocity and the critical bed friction velocity obtained from Shields' diagram for a given size of the sediment particles.

Figure 6 compares the distributions of the non-dimensional bed-friction velocity, $u_{\tau} / u_{b}$, at $\mathrm{t}=32 \mathrm{t}_{\mathrm{o}}$ for the simulations conducted at $\mathrm{Re}=10^{6}$. One obvious feature of the distributions of $u_{\tau} / u_{b}$ is the streaky structure of the region behind the front. The bed-friction-velocity streaks are due to the formation of streaks of high and low streamwise velocity at a small distance from the bed in the region where the flow inside the gravity current is strongly turbulent. The streaks disappear only in the regions situated upstream and downstream of each rib. After the jet-like flow past the top of each rib reattaches to the bed, the streaks need a certain distance to form again beneath the gravity current flow. Then the streaks disappear again as the near-bed flow decelerates close to the upstream face of the next rib. Thus, one expects the formation of the streaks will be suppressed if the spacing of the ribs is sufficiently decreased.

Another relevant feature of the distribution of $u_{\tau} / u_{b}$ in the Flat_HR simulation (Figure 6) is the fact that, with the exception of the head region, there are no regions of strong amplification of $u_{\tau} / u_{b}$ with respect to the mean value $(\sim 0.013)$. This means that in the later stages of the slumping phase $\left(t>20 t_{0}\right)$ the interfacial billows shed in the dissipative wake region are not strong enough to amplify significantly the bed friction velocity values beneath them. This is because the 
cores of the Kelvin Helmholtz billows are strongly disturbed in the formation region, in part due to their interactions with the lobes and the clefts present at the front of the current. This explains why the distribution of the bed friction velocity is relatively uniform behind the front region during the later stages of the slumping phase for a gravity current propagating over a smooth surface. The distributions of $u_{t} / u_{b}$ are qualitatively similar for the Flat_LR simulation but, as expected, the mean value of $u_{\tau} / u_{b}$ is larger $(\sim 0.028)$.

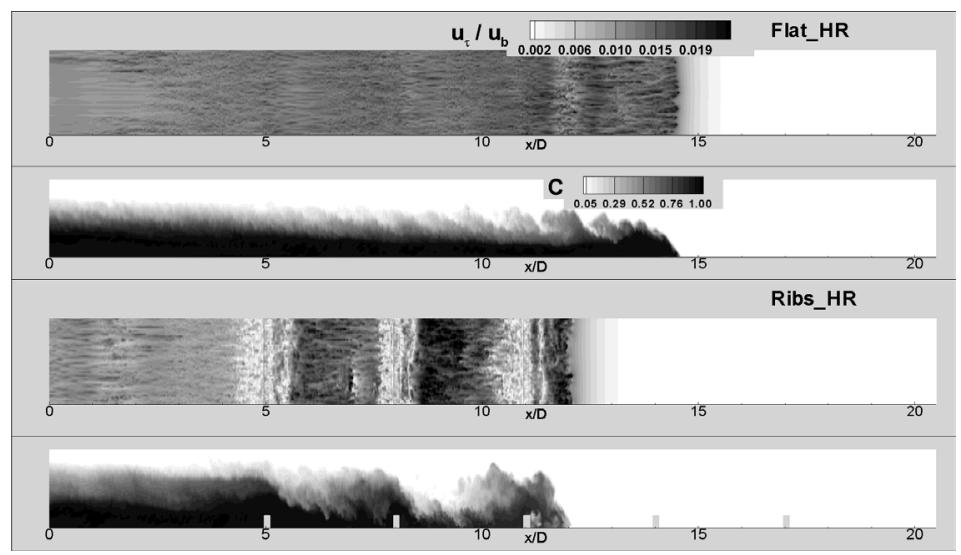

Figure 6: Distribution of the non-dimensional friction velocity, $\mathrm{u}_{\tau \mathrm{c}} / \mathrm{u}_{\mathrm{b}}$, on the bottom wall $(\mathrm{x} / \mathrm{H}>0)$ at $\mathrm{t}=32 \mathrm{t}_{0}$ in the Flat_HR (top) and Ribs_HR (bottom) simulations. Also shown are the corresponding concentration contours (aspect ratio is 1:2) in an $\mathrm{x}-\mathrm{y}$ section.

In the Ribs_HR simulation results, the distribution of $u_{\tau} / u_{b}$ is strongly modulated by the presence of the roughness elements. The bed-friction velocity is strongly amplified downstream of each of the ribs, in the region where the flow inside the gravity current plunges downwards in the form of a jet-like flow and reaches the flat part of the bed surface. After the front passes several ribs, the largest amplification does not always occur downstream of the rib situated the closest to the front, as was the case in the initial stages of the slumping phase. This is because as a result of the interaction between the front and the ribs, the mixing in the head region is very high (see concentration contours for Ribs_HR simulation in Figure 6) and the mean concentration in the head region is smaller than the one in the upstream part of the gravity current (e.g., around the second rib behind the front in Figure 6). The lowest values of the bed-friction velocity are observed in the region situated upstream of each rib, where the heavier gravity current fluid decelerates and increases its potential energy such that it can be convected over the top of the rib.

Figures 7 and 8 allow a direct comparison of the spanwise averaged bed friction velocity among the four simulations in the later stages of the slumping phase $\left(\mathrm{t}=32 \mathrm{t}_{\mathrm{o}}\right)$. The streamwise distributions of the bed friction velocity are qualitatively similar in the low and high Reynolds number simulations. 
However, important differences are observed in the distribution of this variable between the simulations with a flat bed and the simulations with a bed containing ribs. Consistent with the results in Figure 6, the presence of the ribs induces large-scale variations in the streamwise distribution of the spanwise-averaged bed friction velocity. The peak values present in the region where the current reattaches to the flat part of the bed in the simulation containing the ribs are larger than the values observed in the same region in the simulation with a flat bed conducted at the same Reynolds number. For example, the nondimensional peak values of the bed friction velocity behind the head region are around 0.02 in the Ribs_HR simulation, while the corresponding values in the Flat HR simulation are around 0.015 (see Figure 7). The differences are even larger in the simulations conducted at $\mathrm{Re}=48,000$. The peak values in the Ribs_LR simulation are close to 0.045 while the corresponding values in the Flat_LR simulation are around 0.03 (see Figure 8).

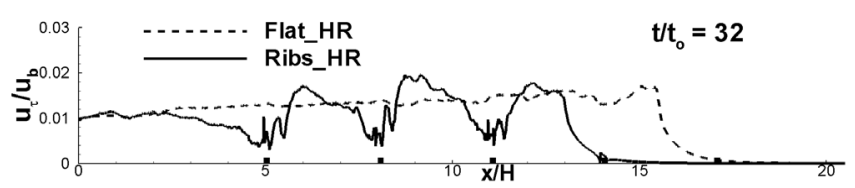

Figure 7: Streamwise variation of the spanwise-averaged friction velocity on the bottom wall $(\mathrm{x} / \mathrm{H}>0)$ at $\mathrm{t}=32 \mathrm{t}_{0}$ in the Flat_HR (dashed line) and Ribs_HR (solid line) simulations.

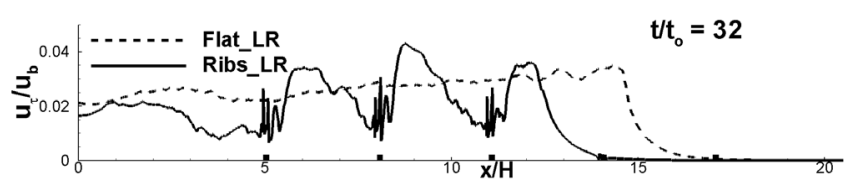

Figure 8: $\quad$ Streamwise variation of the spanwise-averaged friction velocity on the bottom wall $(\mathrm{x} / \mathrm{H}>0)$ at $\mathrm{t}=32 \mathrm{t}_{0}$ in the Flat_LR (dashed line) and Ribs_LR (solid line) simulations.

Based on the results in Figure 7, the mean value of the spanwise-averaged bed friction velocity in between $\mathrm{x} / \mathrm{H}=0.0$ and the front is 0.011 in the Ribs_HR simulation and 0.013 in the Flat_HR simulation. This means that a gravity current propagating over a flat beed in a high Reynolds number $\left(\sim 10^{6}\right)$ lockexchange flow will have a slightly higher capacity to entrain fine sediment compared to one propagating over a bed containing large-scale roughness elements in the form of $2 \mathrm{D}$ ribs. Same trend is observed in the simulations conducted at $\mathrm{Re}=48,000$ where the relative difference between the streamwiseaveraged values in between $\mathrm{x} / \mathrm{H}=0.0$ and the front is similar $(0.026$ in the Flat_LR simulation and 0.021 in the Ribs_LR simulation). Of course, at both Reynolds numbers the capacity of the gravity current to entrain coarser sediment is expected to be higher in the case in which the gravity current propagates over an array of $2 \mathrm{D}$ ribs mainly due to the strong amplification of the bed friction 
velocity in the region where the jet-like flow of heavier fluid reattaches to the bed. While in the flat bed case one expects the capacity of the current to entrain sediment will peak behind the front which is propagating with constant velocity, in the case in which ribs are present the capacity of the current to entrain sediment will be the highest downstream of the first 3-4 ribs behind the front.

To quantitatively compare the capacity of the gravity current to entrain sediment particles of a certain size in the four simulations, the flux of the sediment entrained from the bed per unit time and unit width is calculated for a certain sediment size and Shields critical shear velocity, $u_{\tau c}$, using:

$$
F(t)=\frac{1}{W} \int_{A} P d A
$$

where $\mathrm{W}(=\mathrm{H})$ is the width of the channel, $\mathrm{A}$ is the bed area and $\mathrm{P}$ is the local pick-up rate for the sediment. In the present work the expression proposed by van Rijn (1984) was used to estimate $\mathrm{P}$ when $u_{\tau}>u_{\tau c}$.

$$
P=0.00033\left(\frac{u_{\tau}^{2}-u_{\tau c}^{2}}{u_{\tau c}^{2}}\right)^{1.5} \frac{(s-1)^{0.6} g^{0.6} d^{0.8}}{v^{0.2}}
$$

The pick-up rate $\mathrm{P}$ is expressed in units of volumetric flux per unit area per unit time. If at a certain location $u_{\tau}<u_{\tau c}$, then the local sediment entrainment is equal to zero ( $\mathrm{P}=0)$. In Equation (2), $d$ is the diameter of the sediment, $g$ is the gravitational acceleration, $v$ is the molecular viscosity and $s$ is the ratio between the density of the sediment and that of the fluid.

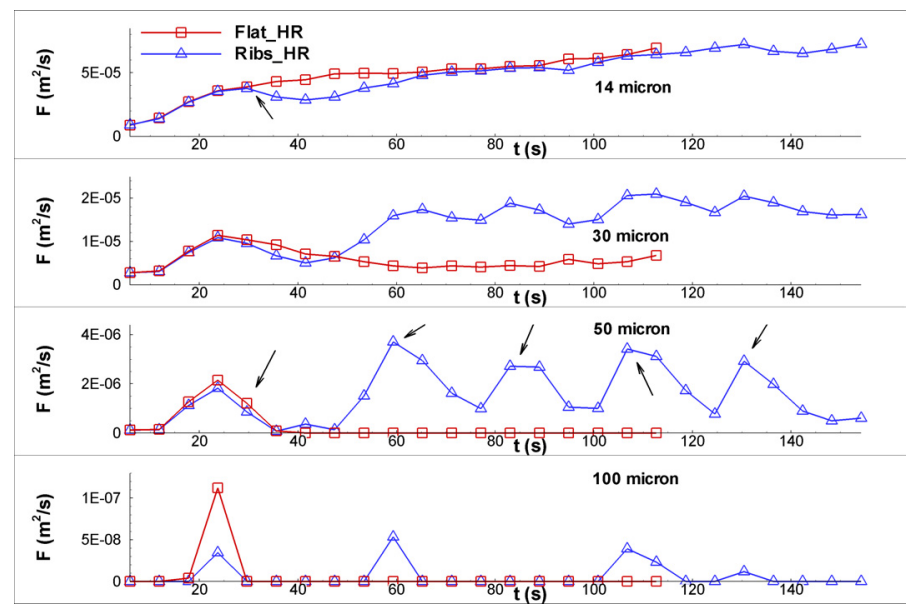

Figure 9: Time history of the flux of sediment entrained at the bed $(\mathrm{x} / \mathrm{H}>0)$ for particles with a threshold value for entrainment of $\mathrm{u}_{\mathrm{\tau c}} / \mathrm{u}_{\mathrm{b}}=0.03$ (top) and $\mathrm{u}_{\tau \mathrm{c}} / \mathrm{u}_{\mathrm{b}}=0.01$ (bottom) in the Flat_LR (circles) and Ribs_LR (squares) simulations.

Assuming a length scale $\mathrm{h}=1.7 \mathrm{~m}$ corresponding to $\Delta \rho / \rho_{0}=0.02$, the buoyancy velocity in the $R e=10^{6}$ simulations is $u_{b}=0.58 \mathrm{~m} / \mathrm{s}$ and $t_{0} \sim 3 \mathrm{~s}$, if the ambient fluid 
is water. The flux $\mathrm{F}(\mathrm{t})$ was plotted in Figure 9 for the simulations conducted at $\mathrm{Re}=10^{6}$ for four sediment sizes $(\mathrm{d}=14,30,50$ and $100 \mu \mathrm{m})$. Using Shields diagram, the critical bed friction velocity $\mathrm{u}_{\tau \mathrm{c}}$ for sediment entrainment is 0.006 , $0.008,0.01,0.012 \mathrm{~m} / \mathrm{s}$ for the four sediment sizes with $\mathrm{d}=14,30,50$ and $100 \mu \mathrm{m}$.

Past the initial stages of the slumping phase $(t>40 \mathrm{~s}$ during which the front of the gravity current passes the first rib in the Ribs_HR simulation) the capacity of the gravity current propagating over ribs to entrain particles with $\mathrm{d}<15$ microns (Figure 9a) is similar to that of the gravity current propagating over a flat bed. For particles with $d=30 \mu \mathrm{m}$ (Figure $9 \mathrm{~b}$ ), the flux of sediment entrained at the bed for $\mathrm{t}>40 \mathrm{~s}$ is three to five times larger for the gravity current propagating over ribs. For $\mathrm{d}>50 \mu \mathrm{m}$ (Figures $9 \mathrm{c}$ and $9 \mathrm{~d}$ ) the gravity current propagating over a flat bed cannot entrain sediment past the initial stages of the slumping phase as $\mathrm{u}_{\tau}<\mathrm{u}_{\tau \mathrm{c}}$. In the case of the gravity current propagating over a bed containing ribs $\mathrm{u}_{\tau}$ remains larger than $\mathrm{u}_{\tau \mathrm{c}}$ in some of the regions where the jet-like flow reattaches downstream of the first couple of ribs behind the front of the current. The flux of coarser sediment is modulated in time by the formation of a region of strong bed friction velocity amplification each time the gravity current overtakes a new rib.

\section{Conclusions}

The erosional properties of high Reynolds number gravity currents with a large volume of release propagating over a flat bed and over a flat bed containing an array of equally-spaced $2 \mathrm{D}$ ribs was investigated for $48,000<\operatorname{Re}<10^{6}$.

Present results show that a gravity current with a high volume of release propagating over an array of $2 \mathrm{D}$ ribs reaches a regime in which the front velocity is nearly constant (slumping phase). The presence of an array of equally spaced $(3 \mathrm{H})$ square $2 \mathrm{D}$ ribs of size $0.15 \mathrm{H}^{*} 0.15 \mathrm{H}$ reduced the velocity of the front by about $24 \%$ at $\mathrm{Re}=48,000$ and by about $27 \%$ at $\mathrm{Re}=10^{6}$ compared to the flat-bed case. In the flat-bed simulation at $\mathrm{Re}=10^{6}$ the predicted non-dimensional front velocity during the slumping phase $(0.49)$ was very close to the value obtained from theory for inviscid currents $(0.5)$.

In the case the ribs were present, the largest bed friction velocity values were observed downstream of the reattachment line of the plunging jet-like flow that forms as the gravity current is convected over the top of the ribs. The largest values did not necessarily occur at all times downstream of the first rib behind the front, especially after the gravity current overtook the first couple of ribs in the series. This is because the heavier lock fluid in the head region mixed with the lighter surrounding fluid. At both Reynolds numbers, the peak bed friction velocity values in the simulations containing $2 \mathrm{D}$ ribs were larger than the ones recorded at the same nondimensional time for the gravity current propagating over a flat bed. This has important consequences in terms of the capacity of the gravity current to entrain coarser material for which the critical bed shear stress value for entrainment is around or above the maximum bed shear stress value predicted in the flat-bed case. 
At both Reynolds numbers, past the initial stages of the slumping phase $\left(t>15 t_{0}\right)$ the mean value of the spanwise-averaged bed friction velocity in between $\mathrm{x} / \mathrm{H}=0.0$ and the front was about $20 \%$ lower in the case the bed contained ribs. However, the presence of the ribs induced the formation of bands of high bed-friction velocity. In these regions larger particles can be entrained by the gravity current. Indeed, analysis of the flux of sediment entrained at the bed in the $\mathrm{Re}=10^{6}$ simulation $\left(\mathrm{h}=1.7 \mathrm{~m}, \Delta \rho / \rho_{0}=0.02, \mathrm{u}_{\mathrm{b}}=0.58 \mathrm{~m} / \mathrm{s}\right)$ past the initial stages of the slumping phase, confirmed that the capacity of the gravity current propagating over a flat bed to entrain fine sediments ( $d<15$ microns) is at least equal to that of the same gravity current propagating over a bed containing ribs. However, for larger particles the capacity to entrain sediment of the compositional gravity current propagating over a flat bed was much smaller than the one estimated for the same current propagating over a bed containing ribs.

\section{References}

[1] Chang, K.S., Constantinescu, G., Park, S-O., Analysis of the flow and mass transfer processes for the incompressible flow past an open cavity with a laminar and a fully turbulent incoming boundary layer. J. Fluid Mech. 561, pp. 113-145, 2006.

[2] Chang, K.S., Constantinescu, G., Park, S-O., The purging of a neutrally buoyant or a dense miscible contaminant from a rectangular cavity. Part II: The case of an incoming fully turbulent overflow. J. Hydraulic Engineering, 133(4), pp. 373-385, 2007.

[3] Härtel, C., Meiburg, E., and Necker, F., Analysis and direct numerical simulation of the flow at a gravity-current head. Part 1: Flow topology and front speed for slip and no-slip boundaries. J. Fluid Mechanics, 418, pp. 189-212, 2000.

[4] Hatcher, L, Hogg, A. and Woods, A., The effect of drag on turbulent gravity currents. J. Fluid Mechanics, 416, pp. 297-314, 2000.

[5] Keulegan, G.H., An experimental study of the motion of saline water from locks into fresh water channels. U.S. Natl. Bur. Stand. Rep. 5168, 1957.

[6] Ooi, S.K., Constantinescu, S.G. and Weber, L., A numerical study of intrusive compositional gravity currents. Physics of Fluids, 19, 076602, 2007.

[7] Shin, J., Dalziel, S., and Linden, P.F., Gravity currents produced by lock exchange. J. Fluid Mech., 521, pp. 1-34, 2004.

[8] Van Rijn, L.C., Sediment pick-up functions. J. Hydraulics Engineering, 110(10), pp. 1494-1503, 1984. 